\title{
Acute effects of cinnamon on glucose response in vivo to a standard carbohydrate-rich food
}

\author{
T. Aldayel ${ }^{1}$, S. M. Hampton ${ }^{2}$, S. A. Lanham-New ${ }^{1}$ and J. E. Brown ${ }^{1}$ \\ ${ }^{1}$ Department of Nutritional Sciences and ${ }^{2}$ Department of Biochemistry and Physiology, Institute of Biosciences and \\ Medicine, Faculty of Health and Medical Sciences, University of Surrey, Guildford, GU2 7XH, UK
}

Many traditional medicinal herbs have been used for the treatment of diabetes mellitus (DM) especially in developing countries ${ }^{(1)}$. Cinnamon has been used for the treatment of DM as it is considered to have hypoglycaemic effects ${ }^{(2)}$. Cinnamon contains many active compounds such as the procyanidins, which may improve glucose levels and insulin sensitivity ${ }^{(3,4)}$. There is evidence that cinnamon polyphenols may decrease the activity of $\alpha$-glucosidase culminating in a slowing of the absorption of glucose in the small intestine ${ }^{(1)}$. The aim of this study was to determine if the ingestion of Cinnamon cassia (C. cassia) supplements, just before the consumption of a carbohydrate-rich food, could reduce starch hydrolysis and therefore affect the glycaemic response in vivo.

A cinnamon supplement (GOODN NATURAL ${ }^{\circledR}$ Cinnamomum cassia, Holland and Barrett Ltd.) was used. New capsules (Vcaps ${ }^{\circledR}$ capsules) were filled with the cinnamon supplement so that the placebo and cinnamon capsules appeared identical. The cinnamon dose used was $1 \mathrm{~g}$. The study design received a favourable ethical opinion (University of Surrey Ethics Committee) and ten healthy participants completed the study. Each participant consumed each test food on two separate occasions after an overnight fast. The cinnamon supplement or placebo capsules were ingested with $100 \mathrm{ml}$ of water $15 \mathrm{~min}$. prior to the test food (cornflakes) (time minus $15 \mathrm{~min}$ ). Then, a portion of cornflakes containing $25 \mathrm{~g}$ of available carbohydrate was served to the participants with $150 \mathrm{ml}$ of water (time $0 \mathrm{~min}$ ). On the three other occasions, the participants consumed the control food (glucose drink), which was $25 \mathrm{~g}$ of glucose in $250 \mathrm{ml}$ of water. Capillary blood samples were collected after an overnight fast $(-15 \mathrm{~min})$, after which time participants were asked to ingest either the cinnamon or placebo capsules. Another blood sample was collected 15 min afterwards (time 0 min). Further samples were taken at $15,30,45,60,90$ and 120 minutes.

No significant differences (Fig. $1 ; p>0.05$ ) were seen in glycaemic response at the different time periods, or in the incremental area under the curve between C.cassia supplement and placebo. Furthermore, cinnamon had no significant effect (Fig.2; $p>0.05)$ on fasting plasma glucose levels compared to placebo.

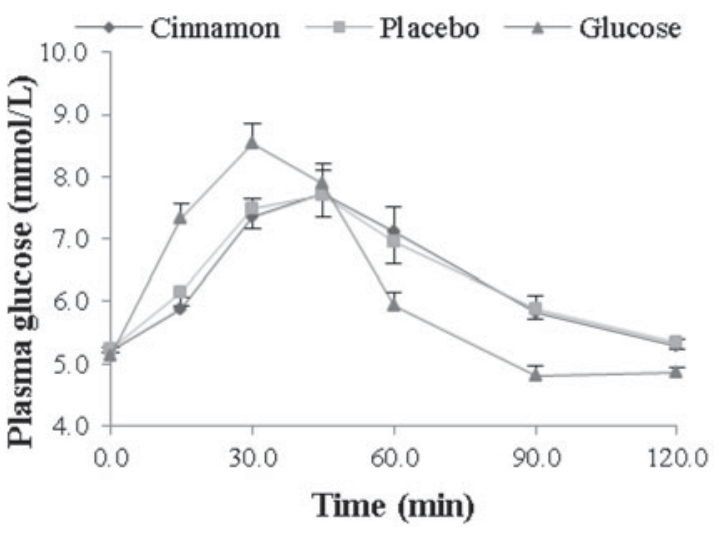

Fig. 1. Glucose response.

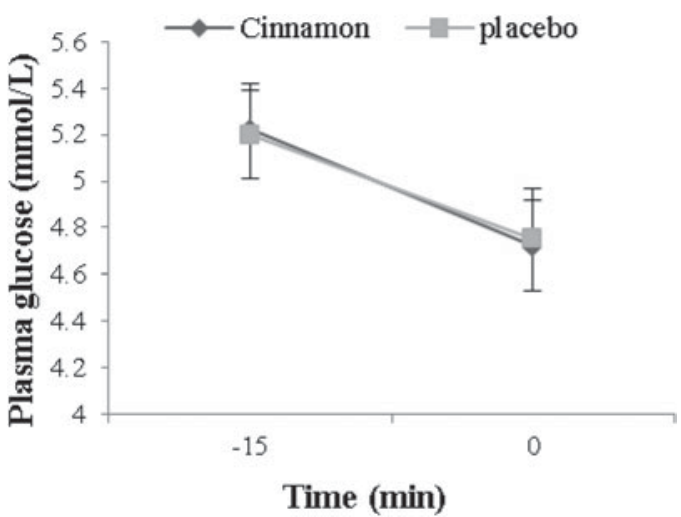

Fig. 2. Fasting plasma glucose level.

Several factors may explain our results: $1 \mathrm{~g}$ of $C$. cassia supplement may have been insufficient to affect the glycaemic response and the time that the cinnamon supplement was present before test food might have not been long enough to alter glucosidase activity and insulin sensitivity. Further research is currently underway to investigate the health benefits of cinnamon at higher doses.

TA is funded by Princess Nora bint Abdulrahman University, Kingdom of Saudi Arabia.

1. Kim SH, Hyun SH \& Choung SY (2006) J Ethnopharmacol 104, 119-123.

2. Subash Babu P, Prabuseenivasan S \& Ignacimuthu S (2007) Phytomedicine 14,15-22.

3. Lu Z, Jia Q, Wang R, Wu X et al. (2011) Phytomedicine 18, 298-302.

4. Anderson RA, Broadhurst CL, Polansky MM et al. (2004) J Agric Food Chem 52, 65-70. 